\title{
Barrier Lyapunov Function-Based Fixed-Time FTC for High-Order Nonlinear Systems with Predefined Tracking Accuracy
}

\section{Xiaolin Wang}

Air Force Engineering University

Jihui Xu

Air Force Engineering University

Maolong Lv ( $\sim$ M.Lyu@tudelft.nl )

Technische Universiteit Delft https://orcid.org/0000-0001-6406-2399

Lei Zhang

Air Force Engineering University

\section{Zilong Zhao}

Delft University of Technology: Technische Universiteit Delft

\section{Research Article}

Keywords: High-order nonlinear systems, Fixed-time stability, High-order tan-type BLF, Predefined tracking accuracy

Posted Date: November 19th, 2021

DOI: https://doi.org/10.21203/rs.3.rs-1056917/v1

License: (c) (1) This work is licensed under a Creative Commons Attribution 4.0 International License. Read Full License 


\title{
Barrier Lyapunov Function-Based Fixed-Time FTC for High-Order Nonlinear Systems with Predefined Tracking Accuracy
}

\author{
Xiaolin Wang · Jihui Xu · Maolong Lv · Lei Zhang • Zilong Zhao
}

Received: date / Accepted: date

\begin{abstract}
This article proposes a fixed-time adaptive fault-tolerant control methodology for a larger class of high-order nonlinear systems subject to full-state constraints and actuator faults. In contrast with the stateof-the-art results, the distinguishing feature of our control design consists in proposing a novel high-order tantype barrier Lyapunov function (BLF) which ensures state variables to be some asymmetric time-varying compact sets under tan-type constraints and expands the application range of tan-type BLF (i.e., from low-order to high-order, from symmetric time-invariant to asymmetric time-varying). Apart from this, the proposed control design ensures the tracking errors converge to specified residual sets within fixed-time and makes the size of the convergence regions of tracking errors adjustable a priori by means of a new BLF-based tuning function and a projection operator. A variable-separable lemma is delicately embedded into the control design to extract the control terms in a linear-like fashion which not only overcomes the difficulty that virtual control signals appear in a non-affine manner, but also solves the problem of actuator faults. Comparative simula-
\end{abstract}

X. Wang $\cdot$ J. Xu $\cdot$ L. Zhang

Department of Equipment Management and UAV Engineering College, Air Force Engineering University, Xi'an, Shaanxi 710051, China

E-mail: wangxiaolin_95@163.com; changan6018@163.com; jxzl80926@163.com

M. Lv $\left.{ }^{(}\right)$

Delft Center for Systems and Control, Delft University of Technology, Mekelweg 2, Delft 2628 CD, The Netherlands

E-mail: M.Lyu@tudelft.nl

Z. Zhao

Faculty of Electrical Engineering, Mathematics and Computer Science, Delft University of Technology, Van Mourik Broekmanweg 6, 2628 XE Delft, The Netherlands

E-mail: Z.Zhao-8@tudelft.nl tions results finally validate the effectiveness of the proposed scheme.

Keywords High-order nonlinear systems · Fixed-time stability · High-order tan-type BLF · Predefined tracking accuracy

\section{Introduction}

Many actual systems can be described as high-order nonlinear systems, such as connected vehicles dynamics (e.g. a tractor with several trailers, etc), synchronous motors, or aircraft wing rock dynamics [1]-[3]. Compared with strict-feedback and pure-feedback systems, high-order nonlinear systems are more general in the sense that positive-odd-integer powers appear in the dynamics [4]-[5]. It is well documented in the literature that high-order nonlinear systems are intrinsically more challenging than strict-feedback and pure-feedback systems, as feedback linearization and backstepping methods fail to work [6]. To handle such problem, the addingone-power-integrator method was successfully proposed in [7] by introducing iteratively one high power integrator instead of a linear one. Despite a number of control problems (i.e. global robust stabilization [8], practical tracking control [9], and asymptotic tracking control [10]) have been solved, the system nonlinearities considered in the above-mentioned results are required to satisfy the growth constraints, i.e., $\left|\psi_{m}(\cdot)\right| \leq\left(\left|\chi_{1}\right|^{r_{1}}+\right.$ $\left.\ldots+\left|\chi_{m}\right|^{r_{m}}\right) \rho_{m}\left(\chi_{1}, \ldots, \chi_{m}\right)$ with $\rho_{m}(\cdot)(1 \leq m \leq n-1)$ known nonnegative smooth functions. To remove this limitation, an intelligent tracking control method was firstly developed in [11] without involving any restrictive growth condition. The same strategy, together with the common Lyapunov function method, was extended to deal with switched high-order nonlinear systems in 
[12]. Most recently, a new error compensation method was further derived in [13] to improve the tracking accuracy. Despite those efforts above, these results rarely focus on the rate of convergence. To be specific, only the exponential convergence of tracking error is guaranteed in the aforementioned works, which reveals the convergence time tends to be infinite.

From a practical perspective, the rate of convergence is of great significance to the transient tracking performance [14]. Recently, the finite-time tracking control for lower/high-order nonlinear systems is investigated in [15]-[18], which makes the tracking error converge into the predefined compact set within a finite time. Nevertheless, the convergence time achieved in [15]-[18] depends on the initial states of the system. This inevitably brings up a problem, that is, the convergence time can not be accurately settled when the initial states of the system are unknown. To solve such problem, the fixed-time control [19]-[21] is proposed skillfully, by which the tracking error can converge into a predefined impact set within a fixed time and its upper bound of convergence time is independent of system initial conditions. The authors in [22] established an adaptive fuzzy feedback control mechanism for uncertain high-order nonlinear systems. However, the above results neglect crucial aspects, such as asymmetric time-varying constraints imposed on state variables, specified tracking accuracy whose importance are explained hereafter.

Because state constraints generally exist in actual systems, ignoring these state constraints may deteriorate system performance and even endangers system safety [23]-[24]. In order to prevent violations of state constraints, barrier Lyapunov function (BLF) is exploited to guarantee the non-violation of state constraints, while ensuring closed-loop stability [25]-[26]. Generally, the commonly seen forms include log-type BLF [27], tan-type BLF [28] and integral-type BLF [29]. Compared with common log-type BLF and integraltype BLF, the tan-type BLF can integrate constrain$\mathrm{t}$ analysis into a general method, which can handle constrained/unconstrained situations [30]-[31]. However, these tan-type BLF constraints are symmetric timeinvariant, which cannot be used to handle asymmetric time-varying cases. To our best knowledge, such issue has not been considered for high-order nonlinear systems in the literatures. In addition, the actuator faults are ubiquitous in engineering applications, which may cause the system instability and performance degradation [32]. Motivated by above observations, the main innovative points of this work are listed below:

1) As opposed to the existing symmetric timeinvariant tan-type BLFs [28], [30]-[31], a new type of high-order tan-type BLF is proposed to guarantee that state variables are confined within some asymmetric time-varying sets all the time, and the initial conditions are inside of corresponding sets.

2) A new BLF-based tuning function and a projection operator are delicately embedded into the control design to make the size of the convergence regions of state tracking errors adjustable in the framework of fixed-time stability.

3) Compared with the fixed-time control methodologies of high-order nonlinear systems [22], which only make the tracking errors converge to a bounded but unknown compact set, the bounds of tracking errors in our case can be predefined a priori. A variable-separable lemma is utilized to extract the virtual control signals and actuator faults in a linear-like manner.

\section{Preliminaries And Problem Formulation}

\subsection{Problem Statement}

Consider the uncertain high-order nonlinear systems with the following form:

$$
\left\{\begin{array}{c}
\dot{\xi}_{i}=\Psi_{i}\left(\bar{\xi}_{i}\right)+\Gamma_{i}\left(\bar{\xi}_{i}\right) \xi_{i+1}^{p_{i}}, \\
\dot{\xi}_{n}=\Psi_{n}(\xi)+\Gamma_{n}(\xi) u^{p_{n}}, \\
y=\xi_{1}, 1 \leq i \leq n-1,
\end{array}\right.
$$

where $y \in \mathbb{R}$ is the system output; $u \in \mathbb{R}$ is the control input (to be designed); $\bar{\xi}_{i}=\left[\xi_{1}, \xi_{2}, \ldots, \xi_{i}\right]^{T} \in \mathbb{R}^{i}$ and $\xi=\left[\xi_{1}, \xi_{2}, \ldots, \xi_{n}\right]^{T} \in \mathbb{R}^{n}$ are the states. For $i=$ $1, \ldots, n, \Psi_{i}(\cdot)$ and $\Gamma_{i}(\cdot)$ are unknown continuous functions, and $p_{i}$ are positive odd integers. In this paper, we consider the system (1) to be the time-varying full state constraints, i.e., $\xi_{i}(t)$ is required to remain in the set $\underline{\varpi}_{c i}(t)<\xi_{i}(t)<\bar{\varpi}_{c i}(t)$, where $\underline{\varpi}_{c i}(t)$ and $\bar{\varpi}_{c i}(t)$ such that $\underline{\varpi}_{c i}(t)<\bar{\varpi}_{c i}(t)$.

The actuator fault is taken into account, which contains the gain fault and bias fault. In line with [32], suppose the actuator output when fault occurs is expressed as

$$
u=\mathcal{H} u_{c}+\eta_{u},
$$

where $\mathcal{H}$ denotes the efficiency factor of the actuator and $\eta_{u}$ represents the unknown time-varying bias fault of the actuator. It is assumed that the efficiency factor $\mathcal{H}$ satisfies $0<\underline{\mathcal{H}}<\mathcal{H}<\overline{\mathcal{H}} \leq 1$, where $\underline{\mathcal{H}}$ and $\overline{\mathcal{H}}$ are known constants. In addition, the bias fault is assumed to be bounded by $\eta_{u} \leq \bar{\eta}$ with $\bar{\eta}$ being the corresponding upper bound.

Without loss of generality, the following standard assumptions from literature are made.

Assumption 1: For a continuously differentiable desired trajectory $y_{r}(t)$, there exists a known positive 
number $y_{0}$, i.e. $\left|y_{r}(t)\right| \leq y_{0}(t),\left|\dot{y}_{r}(t)\right| \leq y_{0}(t)$, moreover $y_{0}(t)<\varpi_{b_{i}}(t)$.

Assumption 2: There exist known real constants $\underline{\Gamma}_{i}>0$ and $\bar{\Gamma}_{i}>0,(1 \leq i \leq n)$ such that $\underline{\Gamma}_{i} \leq \Gamma_{i}(\cdot) \leq$ $\bar{\Gamma}_{i}$.

The control objective is to design a fixed-time singularity-free fault-tolerant controller such that:

(1) The asymmetric and time-varying state constraints under fault-tolerant control are not transgressed;

(2) The tracking errors can converge into the specified compact sets within fixed-time.

The following lemmas are useful for deriving the main results.

Lemma 1 [33]: For any $\chi_{1}, \chi_{2} \in \mathbb{R}$ and positive odd integer $b$, there exist real-valued functions $r_{1}(\cdot, \cdot)$ and $r_{2}(\cdot, \cdot)$ such that

$$
\left(\chi_{1}+\chi_{2}\right)^{b}=\ell\left(\chi_{1}, \chi_{2}\right) \chi_{1}^{b}+v\left(\chi_{1}, \chi_{2}\right) \chi_{2}^{b},
$$

where $\ell\left(\chi_{1}, \chi_{2}\right) \in[\underline{\ell}, \bar{\ell}]$ with $\underline{\ell}=1-d$ and $\bar{\ell}=1+d$, where $d=\sum_{k=1}^{b} \frac{b !}{k !(b-k) !} \frac{b-k}{b} l^{\frac{b}{b-k}}$ is an arbitrary constant taking value in $(0,1)$ for some appropriately smal1 constant $l,\left|v\left(\chi_{1}, \chi_{2}\right)\right| \leq \bar{v}(d)=\sum_{k=1}^{b} \frac{b !}{k !(b-k) !} \frac{k}{b} l^{-\frac{b}{k}}$ with $\bar{v}(d)$ being a positive constant.

Lemma 2 [12]: Let $\zeta_{1} \in \mathbb{R}, \zeta_{2} \in \mathbb{R}$ and $r_{1}$ and $r_{2}$ be positive constants. For any $\varpi>0$, it holds that

$$
\left|\zeta_{1}\right|^{r_{1}}\left|\zeta_{2}\right|^{r_{2}} \leq \frac{r_{1} \varpi\left|\zeta_{1}\right|^{r_{1}+r_{2}}}{r_{1}+r_{2}}+\frac{r_{2} \varpi^{-r_{1} / r_{2}}\left|\zeta_{2}\right|^{r_{1}+r_{2}}}{r_{1}+r_{2}} .
$$

Lemma 3 [20]: Consider the nonlinear system

$$
\dot{x}=f(x(t)), x(0)=x_{0},
$$

if there exists a Lyapunov function $V(x)$ for the system (3) such that

$$
\dot{V}(x) \leq-\alpha V^{p}(x)-\beta V^{q}(x),
$$

where $\alpha, \beta>0 ; p>1 ; 0<q<1$, then the origin of the system (3) is fixed-time stable within

$$
T \leq T_{\max }:=\frac{1}{\alpha(p-1)}+\frac{1}{\beta(1-q)} .
$$

\subsection{Radial Basis Function Neural Network}

In theory, RBFNN can approximate any nonlinear function with arbitrary precision by choosing appropriate parameters [34], which is formulated as

$$
\Psi(\chi)=w^{T} \theta+\delta(\chi),
$$

where $\delta(\chi)$ is the minimum approximation error of the RBFNN, the weight vector is expressed as

$$
w^{*}=\arg \min _{w \subseteq \mathbb{R}^{n}}\left\{\Psi(\chi)-w^{T} \theta\right\},
$$

$\theta=\left[\theta_{1}(\chi), \ldots, \theta_{n}(\chi)\right]^{T}$ represents the basis function vector with

$$
\theta_{i}(\chi)=\exp \left[-\frac{\left(\chi-\iota_{i}\right)^{T}\left(\chi-\iota_{i}\right)}{\aleph_{i}^{T} \aleph_{i}}\right], i=1, \cdots, n,
$$

where $n$ is the number of the RBFNN nodes, $\iota_{i}=$ $\left[\iota_{i 1}, \iota_{i 2}, \ldots, \iota_{i q}\right]^{T}$ and $\aleph_{i}$ are the center and width of the Gaussian function, respectively. It is worth noting that, if the $i$ th node is viewed to be activated, $\theta_{i}(\chi)$ has to be in the compact set $\Omega_{\theta}=\left\{\theta_{i}(\chi) \mid 1>\theta_{i}(\chi) \geq \varepsilon_{\min }>0\right\}$ with $\varepsilon_{\min }$ denoting the user-defined activation threshold. If the value of $x$ is in the active region, i.e., $\left\|\chi-\iota_{i}\right\|^{2}$ $\leq-\aleph_{i}^{T} \aleph_{i} \ln \left(\varepsilon_{\min }\right)$, the node $i$ is called to be activated. Therefore, we can conclude that the smaller the value of $\varepsilon_{\min }$ is, the larger the size of active region is. However, the amount of computation will increase dramatically if the value of $\varepsilon_{\min }$ is chosen very small, which reveals the importance of the selection of $\varepsilon_{\min }$.

\section{Fixed-Time Adaptive Controller Design}

A novel fixed-time singularity-free fault-tolerant control scheme is proposed for high-order nonlinear systems. A $\operatorname{RBFNN} w_{i}^{T} \theta_{i}\left(Z_{i}\right)$ with $w_{i} \in R^{i}, \theta_{i}\left(Z_{i}\right) \in R^{i}$ is utilized to handle the approximation of the unknown function $\bar{f}_{i}\left(Z_{i}\right)$ that will be specified latter. We use $\delta_{i}\left(Z_{i}\right)$ to denote the approximation error which is bounded by $\bar{\delta}$, i.e., $\left|\delta_{i}\left(Z_{i}\right)\right| \leq \bar{\delta}$. By lumping $w_{i}$ and $\theta_{i}\left(Z_{i}\right)$ into the following vectors

$$
\begin{gathered}
\Xi_{i}=\left[w_{i}^{T}, \delta_{i}\left(Z_{i}\right)\right]^{T}, \\
\vartheta_{i}\left(Z_{i}\right)=\left[\theta_{i}^{T}\left(Z_{i}\right), 1\right]^{T},
\end{gathered}
$$

and we further have

$$
\bar{\Psi}_{i}\left(Z_{i}\right)=w_{i}^{T} \theta_{i}\left(Z_{i}\right)+\delta_{i}\left(Z_{i}\right)=\Xi_{i}^{T} \vartheta_{i}\left(Z_{i}\right) .
$$

To quantify the control objective, we define a constant $q>0$ as $q=\max _{1 \leq i \leq n}\left\{p_{i}\right\}$ and consider the following change of coordinates

$$
\left\{\begin{array}{l}
\zeta_{1}=\xi_{1}-y_{r} \\
\zeta_{i}=\xi_{i}-\xi_{i, c}, i=2,3, \ldots, n
\end{array}\right.
$$

where $\xi_{i, c}$ represents the virtual control law which will be specified later. Before performing the controller design, define the following switch functions

$$
\begin{gathered}
\varpi_{i}\left(\zeta_{i}(t)\right)=\left\{\begin{array}{l}
\varpi_{b i}\left(\zeta_{i}(t)\right), \text { if } \zeta_{i}(t)>0, \\
\varpi_{a i}\left(\zeta_{i}(t)\right), \text { if } \zeta_{i}(t) \leq 0 .
\end{array}\right. \\
\psi_{i}\left(\zeta_{i}(t)\right)= \begin{cases}1, \text { if }\left|\zeta_{i}(t)\right|>\beta_{i}, \\
0, \text { if }\left|\zeta_{i}(t)\right| \leq \beta_{i} .\end{cases}
\end{gathered}
$$


$\operatorname{sg}_{i}\left(\zeta_{i}(t)\right)=\left\{\begin{array}{cc}\frac{\zeta_{i}^{q-p_{i}+1}(t)}{\left|\zeta_{i}^{q-p_{i}+1}(t)\right|}, & \text { if }\left|\zeta_{i}(t)\right|>\beta_{i}, \\ \frac{\zeta_{i}^{q-p_{i}+1}(t)}{\left[\beta_{i}^{2}-\zeta_{i}^{2}(t)\right]^{2\left(q-p_{i}+1\right)}+\left|\zeta_{i}^{q-p_{i}+1}(t)\right|}, & \text { if }\left|\zeta_{i}(t)\right| \leq \beta_{i} .\end{array}\right.$

where $\varpi_{i}\left(\zeta_{i}(t)\right)$ is the asymmetric time-varying tracking error constraints, $i \in\{1,2, \ldots, n\} . \varpi_{b i}(t)$ and $\varpi_{a i}(t)$ represents the lower and upper bound of tracking error respectively with $\varpi_{b i}(t)>\beta_{i}>0, \varpi_{a i}(t)<-\beta_{i}<0$, $\varpi_{b i}(t)=\bar{\varpi}_{c i}(t)-y_{r}(t)$ and $\varpi_{a i}(t)=y_{r}(t)-\underline{\varpi}_{c i}(t)$.

From the definitions of (10) and (11), one has

$\operatorname{sg}_{i}\left(\zeta_{i}(t)\right) \psi_{i}\left(\zeta_{i}(t)\right)=\left\{\begin{array}{cl}\frac{\zeta_{i}^{q-p_{i}+1}(t)}{\left|\zeta_{i}^{q-p_{i}+1}(t)\right|}, & \text { if }\left|\zeta_{i}(t)\right|>\beta_{i}, \\ 0, & \text { if }\left|\zeta_{i}(t)\right| \leq \beta_{i},\end{array}\right.$

and

$$
\left[\psi_{i}\left(\zeta_{i}(t)\right)\right]^{m}=\psi_{i}\left(\zeta_{i}(t)\right)
$$

where $m$ represents positive integer.

Step 1: Using (1) and (8), differentiating $\zeta_{1}$ with respect to time yields

$$
\dot{\zeta}_{1}=\Psi_{1}\left(\xi_{1}\right)+\Gamma_{1}\left(\xi_{1}\right) \xi_{2}^{p_{1}}-\dot{y}_{r} .
$$

Consider the high-order tan-type BLF as

$$
\mathfrak{L}_{1}=\frac{2 \varpi_{1}^{q-p_{1}+2}(t)}{\pi\left(q-p_{1}+2\right)} \tan \left(\frac{\pi \zeta_{1}^{q-p_{1}+2}(t)}{2 \varpi_{1}^{q-p_{1}+2}(t)}\right) \psi_{1} .
$$

Remark 1. If $\varpi_{1}(t)$ approaches to infinity, the ter$\mathrm{m} \frac{2 \varpi_{1}^{q-p_{1}+2}(t)}{\pi\left(q-p_{1}+2\right)} \tan \left(\frac{\pi \zeta_{1}^{q-p_{1}+2}(t)}{2 \varpi_{1}^{q-p_{1}+2}(t)}\right)$ will tend to $\frac{\zeta_{1}^{q-p_{1}+2}}{q-p_{1}+2}$, thus the proposed high-order tan-type BLF can deal with both the constrained case and the unconstrained case. The conventional BLF in [25], [27] can only handle the constrained case due to the fact $\lim _{\varpi_{1} \rightarrow \infty} \frac{1}{q-p_{1}+2} \times$ $\log \left(\frac{\varpi_{1}^{q-p_{1}+2}}{\varpi_{1}^{q-p_{1}+2}-\zeta_{1}^{q-p_{1}+2}}\right)=0$.

Remark 2. It is well known that there have been many studies on the tan-type BLF constraint control problem [28], [30]-[31], but they only consider the symmetric time-invariant scenarios. However, due to the influence of faults, asymmetric time-varying constraints are more common in actual systems, so the fault-tolerant control of asymmetric time-varying constraints is more practical. That is the reason why we construct asymmetric time-varying BLFs in our paper.

Remark 3. In (15), the switch function $\psi_{1}$ is applied such that the bounds of steady-state tracking errors can be preset as $\beta_{1}$. By utilizing a smooth switch $\operatorname{sg}_{1}\left(\zeta_{1}(t)\right)$, the chattering phenomenon is effectively eliminated, and thus the transient performance of system is improved.

In view of (14) and (15), the time derivative of $\mathfrak{L}_{1}$ can be expressed as

$$
\begin{aligned}
\dot{\mathfrak{L}}_{1}= & \frac{2 \varpi_{1}^{q-p_{1}+1}(t) \dot{\varpi}_{1}(t)}{\pi} \tan \left(\frac{\pi \zeta_{1}^{q-p_{1}+2}(t)}{2 \varpi_{1}^{q-p_{1}+2}(t)}\right) \psi_{1} \\
& +l_{1}\left(\bar{\Psi}_{1}\left(Z_{1}\right)+\Gamma_{1}\left(\xi_{1}\right) \xi_{2}^{p_{1}}-\frac{\dot{\varpi}_{1}(t)}{\varpi_{1}(t)} \zeta_{1}(t)\right) \psi_{1},
\end{aligned}
$$

where $\bar{\Psi}_{1}\left(Z_{1}\right)=\Psi_{1}\left(\xi_{1}\right)-\dot{y}_{r}, l_{1}=\zeta_{1}^{q-p_{1}+1}(t) \Theta_{1}^{-1}, \Theta_{1}=$ $\cos ^{2}\left(\frac{\pi \zeta_{1}^{q-p_{1}+2}(t)}{2 \varpi_{1}^{q-p_{1}+2}(t)}\right)$ and $Z_{1}=\left[\xi_{1}, \dot{y}_{r}\right]^{T}$. Noting $(7)$, it can be known that (16) can be rewritten as

$$
\begin{aligned}
\dot{\mathfrak{L}}_{1}= & l_{1}\left(\Xi_{1}^{T} \vartheta_{1}\left(Z_{1}\right)+\Gamma_{1}\left(\xi_{1}\right) \xi_{2}^{p_{1}}-\frac{\dot{\varpi}_{1}(t)}{\varpi_{1}(t)} \zeta_{1}(t)\right) \psi_{1} \\
& +\frac{2 \varpi_{1}^{q-p_{1}+1}(t) \dot{\varpi}_{i}(t)}{\pi} \tan \left(\frac{\pi \zeta_{1}^{q-p_{1}+2}(t)}{2 \varpi_{1}^{q-p_{1}+2}(t)}\right) \psi_{1} \\
\leq & \frac{2 \varpi_{1}^{q-p_{1}+1}(t) \dot{\varpi}_{i}(t)}{\pi} \tan \left(\frac{\pi \zeta_{1}^{q-p_{1}+2}(t)}{2 \varpi_{1}^{q-p_{1}+2}(t)}\right) \psi_{1} \\
& +l_{1}\left(\Phi \rho_{1}+\Gamma_{1}\left(\xi_{1}\right) \xi_{2}^{p_{1}}-\frac{\dot{\varpi}_{1}(t)}{\varpi_{1}(t)} \zeta_{1}(t)\right) \psi_{1},
\end{aligned}
$$

where $\Phi=\sqrt{\max \left\{w_{i}^{T} w_{i}+\bar{\delta}^{2}\right\}}, i=1,2, \ldots, n, \rho_{1}=$ $\operatorname{sg}_{1}\left(\zeta_{1}\right) \sqrt{\vartheta_{1}^{T}\left(Z_{1}\right) \vartheta_{1}\left(Z_{1}\right)+\lambda_{0}}$ with $\lambda_{0}>0$ being the parameter to be designed. We are now in the position to handle the term $\xi_{2}^{p_{1}}$ in (17) through Lemma 1 as

$$
\xi_{2}^{p_{1}}=\left(\zeta_{2}+\xi_{2, c}\right)^{p_{1}} \leq \bar{v}_{1}\left|\zeta_{2}^{p_{1}}\right|+\ell_{1} \xi_{2, c}^{p_{1}} .
$$

Substitute (18) into (17), and the derivative of $\mathfrak{L}_{1}$ is

$$
\begin{aligned}
\dot{\mathfrak{L}}_{1} \leq & \frac{2 \varpi_{1}^{q-p_{1}+1}(t) \dot{\varpi}_{1}(t)}{\pi} \tan \left(\frac{\pi \zeta_{1}^{q-p_{1}+2}(t)}{2 \varpi_{1}^{q-p_{1}+2}(t)}\right) \psi_{1} \\
& +l_{1}\left(\Phi \rho_{1}+\Gamma_{1}\left(\xi_{1}\right) \ell_{1} \xi_{2, c}^{p_{1}}-\frac{\dot{\varpi}_{1}(t)}{\varpi_{1}(t)} \zeta_{1}(t)\right) \psi_{1} \\
& +\frac{1}{\Theta_{1}} \bar{\Gamma}_{1}\left(\xi_{1}\right) \bar{v}_{1}\left|\zeta_{1}^{q-p_{1}+1} \zeta_{2}^{p_{1}}\right| \psi_{1} .
\end{aligned}
$$

According to Lemma 1, one has

$$
\begin{aligned}
& \bar{\Gamma}_{1}\left(x_{1}\right) \bar{v}_{1}\left|\zeta_{1}^{q-p_{1}+1} \zeta_{2}^{p_{1}}\right| \psi_{1} \\
\leq & \bar{\Gamma}_{1}\left(x_{1}\right) \bar{v}_{1}\left(\frac{q-p_{1}+1}{q} \phi_{1}^{\frac{q-p_{1}+1}{q}} \zeta_{1}^{q+1}+\frac{p_{1}}{q+1} \phi_{1}^{-\frac{p_{1}}{q+1}} \zeta_{2}^{q+1}\right) \psi_{1} \\
\leq & \bar{\Gamma}_{1}\left(x_{1}\right) \bar{v}_{1}\left(\phi_{1}^{\frac{q-p_{1}+1}{q}} \zeta_{1}^{q+1}+\phi_{1}^{-\frac{p_{1}}{q+1}} \zeta_{2}^{q+1}\right) \psi_{1} .
\end{aligned}
$$

Substitute (20) into (19) yields

$$
\dot{\mathfrak{L}}_{1} \leq \frac{1}{\Theta_{1}} \bar{\Gamma}_{1}\left(\xi_{1}\right) \bar{v}_{1}\left(\phi_{1}^{\frac{q-p_{1}+1}{q}} \zeta_{1}^{q+1}+\phi_{1}^{-\frac{p_{1}}{q+1}} \zeta_{2}^{q+1}\right) \psi_{1}
$$




$$
\begin{aligned}
& +\frac{2 \varpi_{1}^{q-p_{1}+1}(t) \dot{\varpi}_{1}(t)}{\pi} \tan \left(\frac{\pi \zeta_{1}^{q-p_{1}+2}(t)}{2 \varpi_{1}^{q-p_{1}+2}(t)}\right) \psi_{1} \\
& +l_{1}\left(\Phi \rho_{1}+\Gamma_{1}\left(\xi_{1}\right) \ell_{1} \xi_{2, c}^{p_{1}}-\frac{\dot{\varpi}_{1}(t)}{\varpi_{1}(t)} \zeta_{1}(t)\right) \psi_{1} .
\end{aligned}
$$

Design the singularity-free switching controller $\xi_{2, c}$ as

$$
\begin{aligned}
\xi_{2, c}= & -\left[\frac { 1 } { \underline { \Gamma } _ { 1 } ( \xi _ { 1 } ) \underline { \ell } _ { 1 } } \left[\frac{a_{1}}{l_{1}} \tan ^{p}\left(\frac{\pi \zeta_{1}^{q-p_{1}+2}(t)}{2 \varpi_{1}^{q-p_{1}+2}(t)}\right) \operatorname{sg}_{1}\left(\zeta_{1}\right)\right.\right. \\
& +\left(\beta_{2}+1\right) \operatorname{sg}_{1}\left(\zeta_{1}\right)+\frac{1}{l_{1} \Theta_{1}} \bar{\Gamma}_{1}\left(\xi_{1}\right) \bar{v}_{1} \phi_{1}^{\frac{q-p_{1}+1}{q}} \zeta_{1}^{q+1} \\
& +\frac{2 \varpi_{1}^{q-p_{1}+1}(t) \dot{\varpi}_{1}(t)}{l_{1} \pi} \tan \left(\frac{\pi \zeta_{1}^{q-p_{1}+2}(t)}{2 \varpi_{1}^{q-p_{1}+2}(t)}\right) \\
& +\hbar_{1} \zeta_{1}+\frac{c_{1}}{l_{1}} \tan ^{q}\left(\frac{\pi \zeta_{1}^{q-p_{1}+2}(t)}{2 \varpi_{1}^{q-p_{1}+2}(t)}\right) \operatorname{sg}_{1}\left(\zeta_{1}\right) \\
& \left.\left.+\hat{\Phi} \rho_{1}+\frac{l_{1}}{4} \operatorname{sg}_{1}\left(\zeta_{1}\right)+\kappa^{2} \rho_{1} \gamma_{1}\right]\right]^{\frac{1}{p_{1}}},
\end{aligned}
$$

where $c_{1}, a_{1}, \kappa>0$ are the parameters to be designed. The time-varying gain term $\hbar_{1}$ is chosen as

$$
\hbar_{1}=\sqrt{\left(\frac{\dot{\varpi}_{b 1}}{\varpi_{b 1}}\right)^{2}+\left(\frac{\dot{\varpi}_{a 1}}{\varpi_{a 1}}\right)^{2}+o_{1}}
$$

in which $o_{1}>0$ is the parameter to be designed. The switched tuning function $\gamma_{1}$ is chosen as

$\gamma_{1}=\left\{\begin{array}{cc}\tan ^{q}\left(\frac{\pi \zeta_{1}^{q-p_{1}+2}(t)}{2 \varpi_{1}^{q-p_{1}+2}(t)}\right) \rho_{1} \operatorname{sg}_{1}\left(\zeta_{1}\right), & \text { if } \\ 0, & \left|\zeta_{1}(t)\right|>\beta_{1}, \\ \text { if } & \left|\zeta_{1}(t)\right| \leq \beta_{1} .\end{array}\right.$

Remark 4. In (22), it has $\sin ^{q}\left(\frac{\pi \zeta_{1}^{q-p_{1}+2}(t)}{2 \varpi_{1}^{q-p_{1}+2}(t)}\right) \sim\left(\frac{\pi \zeta_{1}^{q-p_{1}+2}(t)}{2 \varpi_{1}^{q-p_{1}+2}(t)}\right)^{q}$ when $\zeta_{1} \rightarrow 0$. According to the L'Hospital's rule, one has

$$
\begin{aligned}
\lim _{\zeta_{1} \rightarrow 0} \frac{\tan ^{q}\left(\frac{\pi \zeta_{1}^{q-p_{1}+2}}{\left.2 \varpi_{1}^{q-p_{1}+2}\right)}\right)}{l_{1}} & =\lim _{\zeta_{1} \rightarrow 0} \frac{\sin ^{q}\left(\frac{\pi \zeta_{1}^{q-p_{1}+2}}{2 \varpi_{1}^{q-p_{1}+2}}\right) \cos ^{2-q}\left(\frac{\pi \zeta_{1}^{q-p_{1}+2}}{\left.2 \varpi_{1}^{q-p_{1}+2}\right)}\right)}{\zeta_{1}^{q-p_{1}+2}} \\
& =\lim _{\zeta_{1} \rightarrow 0} \frac{\left(\frac{\pi \zeta_{1}^{q-p_{1}+2}}{2 \varpi_{1}^{q-p_{1}+2}}\right)^{q}}{\zeta_{1}^{q-p_{1}+2}}=0,
\end{aligned}
$$

that is to say, there is no singularity problem if $\varpi_{1}(t) \neq$ 0 in our proposed fixed-time controller. Furthermore, we note that the inequation $\tan \left(\frac{\pi \zeta_{1}^{q-p_{1}+2}}{2 \varpi_{1}^{q-p_{1}+2}}\right)>0$ holds since $\zeta_{1}<\varpi_{1}$, thus it is feasible to design the tuning function (24).

In view of (23), it can be derived that

$$
-l_{1} \hbar_{1} \zeta_{1}-l_{1} \zeta_{1}(t) \frac{\dot{k}_{1}(t)}{k_{1}(t)}=-\left(\hbar_{1}+\frac{\dot{k}_{1}(t)}{k_{1}(t)}\right) \frac{\zeta_{1}^{q-p_{1}+2}}{\Theta_{1}}<0 .
$$

Substituting (22) and (25) into (21) reaches

$$
\begin{aligned}
\dot{\mathfrak{L}}_{1} \leq & -c_{1} \tan ^{q}\left(\frac{\pi \zeta_{1}^{q-p_{1}+2}(t)}{2 \varpi_{1}^{q-p_{1}+2}(t)}\right) \psi_{1}-l_{1}\left(\beta_{2}+1\right) \psi_{1} \\
& -a_{1} \tan ^{p}\left(\frac{\pi \zeta_{1}^{q-p_{1}+2}(t)}{2 \varpi_{1}^{q-p_{1}+2}(t)}\right) \psi_{1}+\tilde{\Phi} \gamma_{1}-\kappa^{2} \gamma_{1}^{2} \\
& +\frac{1}{\Theta_{1}} \bar{\Gamma}_{1}\left(\xi_{1}\right) \bar{v}_{1} \phi_{1}^{-\frac{p_{1}}{q+1}} \zeta_{2}^{q+1} \psi_{1}-\frac{l_{1}^{2}}{4} \psi_{1}
\end{aligned}
$$

Step $i(i \in\{2, \ldots, n-1\})$ : The design process for step $i$ follows recursively from step 1. From (1) and (8), the time derivative of variable $\zeta_{i}$ can be written as

$$
\dot{\zeta}_{i}=\bar{\Psi}_{i}\left(Z_{i}\right)+\Gamma_{i}\left(\bar{\xi}_{i}\right) \xi_{i+1}^{p_{i}}-\frac{\partial \xi_{i, c}}{\partial \hat{\Phi}} \dot{\hat{\Phi}}
$$

where $\bar{\Psi}_{i}\left(Z_{i}\right)=\Psi_{i}\left(\bar{\xi}_{i}\right)-\sum_{j=1}^{i-1} \frac{\partial \xi_{j+1, c}}{\partial \xi_{j}} \dot{\xi}_{j}-\frac{\partial \xi_{i, c}}{\partial y_{r}} \dot{y}_{r}$ and $Z_{i}=$ $\left[\xi_{1}, \xi_{2}, \ldots, \xi_{i}, \dot{y}_{r}\right]^{T}$. Consider the high-order tan-type BLF as follows

$$
\mathfrak{L}_{i}=\mathfrak{L}_{i-1}+\frac{2 \varpi_{i}^{q-p_{i}+2}(t)}{\pi\left(q-p_{i}+2\right)} \tan \left(\frac{\pi \zeta_{i}^{q-p_{i}+2}(t)}{2 \varpi_{i}^{q-p_{i}+2}(t)}\right) \psi_{i} .
$$

Let $l_{i}=\frac{\zeta_{i}^{q-p_{i}+1}(t)}{\Theta_{i}}$ and $\Theta_{i}=\cos ^{2}\left(\frac{\pi \zeta_{i}^{q-p_{i}+2}(t)}{2 \varpi_{i}^{q-p_{i}+2}(t)}\right)$, then the time derivative of $\mathfrak{L}_{i}$ is

$$
\begin{aligned}
& \dot{\mathfrak{L}}_{i}=l_{i}\left(\Xi_{i}^{T} \vartheta_{i}\left(Z_{i}\right)+\Gamma_{i}\left(\bar{\xi}_{i}\right) \xi_{i+1}^{p_{i}}-\frac{\partial \xi_{i, c}}{\partial \hat{\Phi}} \dot{\hat{\Phi}}-\frac{\dot{\varpi}_{i}(t)}{\varpi_{i}(t)} \zeta_{i}(t)\right) \psi_{i} \\
& +\dot{\mathfrak{L}}_{i-1}+\frac{2 \varpi_{i}^{q-p_{i}+1}(t) \dot{\varpi}_{i}(t)}{\pi} \tan \left(\frac{\pi \zeta_{i}^{q-p_{i}+2}(t)}{2 \varpi_{i}^{q-p_{i}+2}(t)}\right) \psi_{i} \\
& \leq \dot{\mathfrak{L}}_{i-1}+l_{i}\left(\Phi \rho_{i}+\Gamma_{i}\left(\bar{\xi}_{i}\right) \xi_{i+1}^{p_{i}}-\frac{\partial \xi_{i, c}}{\partial \hat{\Phi}} \dot{\hat{\Phi}}-\frac{\dot{\varpi}_{i}(t)}{\varpi_{i}(t)} \zeta_{i}(t)\right) \psi_{i} \\
& +\frac{2 \varpi_{i}^{q-p_{i}+1}(t) \dot{\varpi}_{i}(t)}{\pi} \tan \left(\frac{\pi \zeta_{i}^{q-p_{i}+2}(t)}{2 \varpi_{i}^{q-p_{i}+2}(t)}\right) \psi_{i},
\end{aligned}
$$

where $\rho_{i}=\operatorname{sg}_{i}\left(\zeta_{i}\right) \sqrt{\vartheta_{i}^{T}\left(Z_{i}\right) \vartheta_{i}\left(Z_{i}\right)+\lambda_{0}}$ with $\lambda_{0}>0$ being the parameter to be designed. Along similar lines as (18)-(20), one has

$$
\begin{aligned}
\dot{\mathfrak{L}}_{i} \leq & l_{i}\left(\Phi \rho_{i}+\Gamma_{i}\left(\bar{\xi}_{i}\right) \ell_{i} \xi_{i+1, c}^{p_{i}}-\frac{\dot{\varpi}_{i}(t)}{\varpi_{i}(t)} \zeta_{i}(t)-\frac{\partial \xi_{i, c}}{\partial \hat{\Phi}}\right) \psi_{i} \\
& +\dot{\mathfrak{L}}_{i-1}+\frac{2 \varpi_{i}^{q-p_{i}+1}(t) \dot{\varpi}_{i}(t)}{\pi} \tan \left(\frac{\pi \zeta_{i}^{q-p_{i}+2}(t)}{2 \varpi_{i}^{q-p_{i}+2}(t)}\right) \psi_{i} \\
& +\frac{1}{\Theta_{i}} \bar{\Gamma}_{i}\left(\bar{\xi}_{i}\right) \bar{v}_{i}\left(\phi_{i}^{\frac{q-p_{i}+1}{q}} \zeta_{i}^{q+1}+\phi_{i}^{-\frac{p_{i}}{q+1}} \zeta_{i+1}^{q+1}\right) \psi_{i} .
\end{aligned}
$$

Design the singularity-free switching controller $\xi_{i+1, c}$ as $\xi_{i+1, c}=-\left[\frac{1}{\underline{\Gamma}_{i}\left(\bar{\xi}_{i}\right) \underline{\ell}_{i}}\left[\frac{1}{l_{i} \psi_{i} \Theta_{i-1}} \bar{\Gamma}_{i-1}\left(\bar{\xi}_{i-1}\right) \bar{v}_{i-1} \phi_{i-1}^{-\frac{p_{i-1}}{q+1}} \zeta_{i}^{q+1} \psi_{i-1}\right.\right.$ 


$$
\begin{aligned}
& +\frac{c_{i}}{l_{i}} \tan ^{q}\left(\frac{\pi \zeta_{i}^{q-p_{i}+2}(t)}{2 \varpi_{i}^{q-p_{i}+2}(t)}\right) \operatorname{sg}_{i}\left(\zeta_{i}\right)+\frac{1}{l_{i}} \beta_{i}^{2} \psi_{i} \operatorname{sg}_{i}\left(\zeta_{i}\right) \\
& +\frac{a_{i}}{l_{i}} \tan ^{p}\left(\frac{\pi \zeta_{i}^{q-p_{i}+2}(t)}{2 \varpi_{i}^{q-p_{i}+2}(t)}\right) \operatorname{sg}_{i}\left(\zeta_{i}\right)+\kappa^{2} \rho_{i}\left(\gamma_{i-1}+\gamma_{i}\right) \\
& +\hat{\Phi} \rho_{i}+\hbar_{i} \zeta_{i}+\frac{l_{i}}{4} \operatorname{sg}_{i}\left(\zeta_{i}\right)+\frac{1}{l_{i} \Theta_{i}} \bar{\Gamma}_{i}\left(\bar{\xi}_{i}\right) \bar{v}_{i} \phi_{i}^{\frac{q-p_{i}+1}{q}} \zeta_{i}^{q+1} \\
& +\frac{2 \varpi_{i}^{q-p_{i}+1}(t) \dot{\varpi}_{i}(t)}{l_{i} \pi} \tan \left(\frac{\pi \zeta_{i}^{q-p_{i}+2}(t)}{2 \varpi_{i}^{q-p_{i}+2}(t)}\right) \psi_{i} \\
& \left.\left.+\left(\beta_{i+1}+1\right) \operatorname{sg}_{i}\left(\zeta_{i}\right)+\frac{l_{i}}{4} \operatorname{sg}_{i}\left(\zeta_{i}\right)\left(\frac{\partial \xi_{i, c}}{\partial \hat{\Phi}}\right)^{2}\right]\right]^{\frac{1}{p_{i}}}
\end{aligned}
$$

here the time-varying gain term $\hbar_{i}$ is chosen as

$$
\hbar_{i}=\sqrt{\left(\frac{\dot{\varpi}_{b i}}{\varpi_{b i}}\right)^{2}+\left(\frac{\dot{\varpi}_{a i}}{\varpi_{a i}}\right)^{2}+o_{i}}
$$

in which $o_{i}>0$ is the parameter to be designed. The switched tuning function $\gamma_{i}$ is chosen as

$\gamma_{i}=\left\{\begin{array}{cc}\tan ^{q}\left(\frac{\pi \zeta_{i}^{q-p_{i}+2}(t)}{2 \varpi_{i}^{q-p_{i}+2}(t)}\right) \rho_{i} \operatorname{sg}_{i}\left(\zeta_{i}\right)+\gamma_{i-1}, & \text { if }\left|\zeta_{i}(t)\right|>\beta_{i}, \\ \gamma_{i-1}, & \text { if }\left|\zeta_{i}(t)\right| \leq \beta_{i} .\end{array}\right.$

Substituting (31) to (30) and using the fact that $-l_{i} \hbar_{i} \zeta_{i}-$ $l_{i} \zeta_{i}(t) \frac{\dot{\varpi}_{i}(t)}{\varpi_{i}(t)}=-\left(\hbar_{i}+\frac{\dot{\varpi}_{i}(t)}{\varpi_{i}(t)}\right) \frac{\zeta_{i}^{q-p_{i}+2}}{\Theta_{i}}<0$, we can further have

$$
\begin{aligned}
\dot{\mathfrak{L}}_{i} \leq & -\sum_{m=1}^{i} c_{m} \tan ^{q}\left(\frac{\pi \zeta_{m}^{q-p_{m}+2}(t)}{2 \varpi_{m}^{q-p_{m}+2}(t)}\right) \psi_{m}+\tilde{\Phi} \gamma_{i}-\frac{l_{i}^{2}}{4} \psi_{i} \\
& -\sum_{m=1}^{i} a_{m} \tan ^{p}\left(\frac{\pi \zeta_{m}^{q-p_{m}+2}(t)}{2 \varpi_{m}^{q-p_{m}+2}(t)}\right) \psi_{m}+\wp_{i}+\Im_{i} \\
& +l_{i}\left(-\beta_{i+1}-1\right) \psi_{i}+\frac{1}{\Theta_{i}} \bar{\Gamma}_{i}\left(\bar{\xi}_{i}\right) \bar{v}_{i} \phi_{i}^{-\frac{p_{i}}{q+1}} \zeta_{i+1}^{q+1} \psi_{i},
\end{aligned}
$$

where

$$
\begin{aligned}
\wp_{i}= & -\frac{l_{i-1}^{2}}{4} \psi_{i-1}+l_{i-1}\left(-\beta_{i}-1\right) \psi_{i-1}-\beta_{i}^{2} \psi_{i} \\
\Im_{i}= & -\kappa^{2} \gamma_{i}^{2}-\sum_{m=2}^{i} l_{m} \psi_{m} \operatorname{sg}_{m}\left(\zeta_{m}\right) \frac{\partial \xi_{m+1, c}}{\partial \hat{\Phi}} \dot{\hat{\Phi}} \\
& -\sum_{m=2}^{i} \frac{l_{m}^{2}}{4} \psi_{m}\left(\frac{\partial \xi_{m+1, c}}{\partial \hat{\Phi}}\right)^{2} .
\end{aligned}
$$

It should be worth noting that $\wp_{i}<0$ is always satisfied. If $0 \leq \beta_{i}+1$, the inequation $l_{i-1}\left(-\beta_{i}-1\right) \psi_{i-1} \leq 0$ holds, if $0>\beta_{i}+1$, the inequation $l_{i-1}\left(-\beta_{i}-1\right) \psi_{i-1} \leq$ $\frac{l_{i-1}^{2}}{4} \psi_{i-1}+\left(-\beta_{i}-1\right)^{2} \leq \frac{l_{i-1}^{2}}{4} \psi_{i-1}+\beta_{i}^{2} \psi_{i}$ holds and thus $\wp_{i}<0$.
Step $n$ : Invoking (1) and (8), the dynamics of $\dot{\zeta}_{n}$ are given by

$$
\dot{\zeta}_{n}=\bar{\Psi}_{n}\left(Z_{n}\right)+\Gamma_{n}(\xi) \ell_{n} \mathcal{H}^{p_{n}} u_{c}^{p_{n}}-\frac{\partial \xi_{i, c}}{\partial \hat{\Phi}} \dot{\hat{\Phi}}
$$

where $\bar{\Psi}_{n}\left(Z_{n}\right)=\Psi_{n}(\xi)-\sum_{i=1}^{n-1} \frac{\partial \xi_{i+1, c}}{\partial \xi_{i}} \dot{\xi}_{i}-\frac{\partial \xi_{n, c}}{\partial y_{r}} \dot{y}_{r}+\Gamma_{n}(\xi) v_{n} \eta_{u}^{p_{n}}$ and $Z_{n}=\left[\xi_{1}, \xi_{2}, \ldots, \xi_{n}, \dot{y}_{r}\right]^{T}$. Take the high-order tantype BLF as

$$
\begin{aligned}
\mathfrak{L}_{n}= & \mathfrak{L}_{n-1}+\frac{2 \varpi_{n}^{q-p_{n}+2}(t)}{\pi\left(q-p_{n}+2\right)} \tan \left(\frac{\pi \zeta_{n}^{q-p_{n}+2}(t)}{2 \varpi_{n}^{q-p_{n}+2}(t)}\right) \psi_{n} \\
& +\frac{\tilde{\Phi}^{2}}{2 \kappa} .
\end{aligned}
$$

Similarly to step $i$, define $l_{n}=\frac{\zeta_{n}^{q-p_{n}+1}(t)}{\Theta_{n}}$ and $\Theta_{n}=$ $\cos ^{2}\left(\frac{\pi \zeta_{n}^{q-p_{n}+2}(t)}{2 \varpi_{n}^{q-p_{n}+2}(t)}\right)$, the time derivative of $\mathfrak{L}_{n}$ can be given by

$$
\begin{aligned}
\dot{\mathfrak{L}}_{n}= & \dot{\mathfrak{L}}_{n-1}+\frac{2 \varpi_{n}^{q-p_{n}+1}(t) \dot{\varpi}_{n}(t)}{\pi} \tan \left(\frac{\pi \zeta_{n}^{q-p_{n}+2}(t)}{2 \varpi_{n}^{q-p_{n}+2}(t)}\right) \psi_{n} \\
& +l_{n}\left(\Xi_{n}^{T} \vartheta_{n}+\Gamma_{n}(\xi) \ell_{n} \mathcal{H}^{p_{n}} u_{c}^{p_{n}}-\frac{\left.\partial \xi_{i, c} \dot{\hat{\Phi}}\right)}{\partial \hat{\Phi}} \psi_{n}\right. \\
& -l_{n} \frac{\dot{\varpi}_{n}(t)}{\varpi_{n}(t)} \zeta_{n}(t) \psi_{n}-\frac{\tilde{\Phi} \dot{\hat{\Phi}}}{\kappa} \\
\leq & \dot{\mathfrak{L}}_{n-1}+\frac{2 \varpi_{n}^{q-p_{n}+1}(t) \dot{\varpi}_{n}(t)}{\pi} \tan \left(\frac{\pi \zeta_{n}^{q-p_{n}+2}(t)}{2 \varpi_{n}^{q-p_{n}+2}(t)}\right) \psi_{n} \\
& +l_{n}\left(\Phi \rho_{n}+\Gamma_{n}(\xi) \ell_{n} \mathcal{H}^{p_{n}} u_{c}^{p_{n}}-\frac{\partial \xi_{i, c} \dot{\hat{\Phi}}}{\partial \hat{\Phi}}\right) \psi_{n} \\
& -l_{n} \frac{\dot{\varpi}_{n}(t)}{\varpi_{n}(t)} \zeta_{n}(t) \psi_{n}-\frac{\tilde{\Phi} \dot{\hat{\Phi}}}{\kappa},
\end{aligned}
$$

where $\rho_{n}=\operatorname{sg}_{n}\left(\zeta_{n}\right) \sqrt{\vartheta_{n}^{T}\left(Z_{n}\right) \vartheta_{n}\left(Z_{n}\right)+\lambda_{0}}$ with $\lambda_{0}>$ 0 being the parameter to be designed. Design the singularityfree switching fault tolerant controller $u_{c}$ and adaptation law $\hat{\Phi}$ as

$$
\begin{aligned}
u_{c}= & -\left[\frac { 1 } { \underline { \Gamma } _ { n } ( \xi ) \underline { \ell } _ { n } \underline { \mathcal { H } } ^ { p _ { n } } } \left[\frac{c_{n}}{l_{n}} \tan ^{q}\left(\frac{\pi \zeta_{n}^{q-p_{n}+2}(t)}{2 \varpi_{n}^{q-p_{n}+2}(t)}\right) \operatorname{sg}_{n}\left(\zeta_{n}\right)\right.\right. \\
& +\hat{\Phi} \rho_{n}+\frac{1}{l_{n} \psi_{n} \Theta_{n-1}} \bar{\Gamma}_{n-1}\left(\bar{\xi}_{n-1}\right) \bar{v}_{n-1} \phi_{n-1}^{-\frac{p_{n-1}+1}{q+1}} \zeta_{n}^{q+1} \psi_{n-1} \\
& +\frac{a_{n}}{l_{n}} \tan ^{p}\left(\frac{\pi \zeta_{n}^{q-p_{n}+2}(t)}{2 \varpi_{n}^{q-p_{n}+2}(t)}\right) \operatorname{sg}_{n}\left(\zeta_{n}\right)+\frac{1}{l_{n}} \beta_{n}^{2} \psi_{n} \operatorname{sg}_{n}\left(\zeta_{n}\right) \\
& +\frac{2 \varpi_{n}^{q-p_{n}+1}(t) \dot{\varpi}_{n}(t)}{l_{n} \pi} \tan \left(\frac{\pi \zeta_{n}^{q-p_{n}+2}(t)}{2 \varpi_{n}^{q-p_{n}+2}(t)}\right) \psi_{n}+\hbar_{n} \zeta_{n} \\
& \left.\left.+\frac{l_{n}}{4} \operatorname{sg}_{n}\left(\zeta_{n}\right)\left(\frac{\partial \xi_{n, c}}{\partial \hat{\Phi}}\right)^{2}+\kappa^{2} \rho_{n}\left(\gamma_{n-1}+\gamma_{n}\right)\right]\right]^{\frac{1}{p_{n}}}, \quad \\
& \dot{\hat{\Phi}}=\operatorname{Proj}\left(\kappa \gamma_{n}\right)=\left\{\begin{array}{cc}
\kappa \gamma_{n}, & \text { if }\left|\zeta_{n}(t)\right|>\beta_{n}, \\
0, & \text { if }\left|\zeta_{n}(t)\right| \leq \beta_{n} .
\end{array}\right.
\end{aligned}
$$


where the time-varying gain term $\hbar_{n}$ is chosen as

$$
\hbar_{n}=\sqrt{\left(\frac{\dot{\varpi}_{b n}}{\varpi_{b n}}\right)^{2}+\left(\frac{\dot{\varpi}_{a n}}{\varpi_{a n}}\right)^{2}+o_{n}}
$$

in which $o_{n}>0$ is the parameter to be designed. The switched tuning function is chosen as

$\gamma_{n}=\left\{\begin{array}{cc}\tan ^{q}\left(\frac{\pi \zeta_{n}^{q-p_{n}+2}(t)}{2 \varpi_{n}^{q-p_{n}+2}(t)}\right) \rho_{n} \operatorname{sg}_{n}\left(\zeta_{n}\right)+\gamma_{n-1}, & \text { if }\left|\zeta_{n}(t)\right|>\beta_{n}, \\ \gamma_{n-1}, & \text { if }\left|\zeta_{n}(t)\right| \leq \beta_{n} .\end{array}\right.$

Using (34) and substituting (39) and (40) into (38) yields

$$
\begin{aligned}
\dot{\mathfrak{L}}_{n} \leq & \frac{l_{n} \psi_{n}}{\tan ^{q}\left(\frac{\pi \zeta_{n}^{q-p_{n}+2}(t)}{2 \varpi_{n}^{q-p_{n}+2}(t)}\right)} \frac{\tilde{\Phi}}{\kappa}\left[\kappa \gamma_{n}-\operatorname{Proj}\left(\kappa \gamma_{n}\right)\right]+\wp_{n} \\
& -\sum_{i=1}^{n} a_{i} \tan ^{p}\left(\frac{\pi \zeta_{i}^{q-p_{i}+2}(t)}{2 \varpi_{i}^{q-p_{i}+2}(t)}\right) \psi_{i}+\Im_{n} \\
& -\sum_{i=1}^{n} c_{i} \tan ^{q}\left(\frac{\pi \zeta_{i}^{q-p_{i}+2}(t)}{2 \varpi_{i}^{q-p_{i}+2}(t)}\right) \psi_{i}
\end{aligned}
$$

where

$$
\begin{aligned}
\wp_{n}= & -\frac{l_{n-1}^{2}}{4} \psi_{n-1}+l_{n-1}\left(-\beta_{n}-1\right) \psi_{n-1}-\beta_{n}^{2} \psi_{n} \\
\Im_{n}= & -\kappa^{2} \gamma_{n}^{2}-\sum_{i=2}^{n} \frac{l_{i}^{2}}{4} \psi_{i} \operatorname{sg}_{i}\left(\zeta_{i}\right)\left(\frac{\partial \xi_{i, c}}{\partial \hat{\Phi}}\right)^{2} \\
& -\sum_{i=2}^{n} l_{i} \psi_{i} \operatorname{sg}_{i}\left(\zeta_{i}\right) \frac{\partial \xi_{i, c}}{\partial \hat{\Phi}} \dot{\hat{\Phi}}
\end{aligned}
$$

According to the property of projection operator (40), we have $\frac{\tilde{\Phi}}{\kappa}\left[\kappa \gamma_{n}-\operatorname{Proj}\left(\kappa \gamma_{n}\right)\right] \leq 0$. With the addition of the fact $\frac{l_{n} \psi_{n}}{\tan ^{q}\left(\frac{\pi \zeta_{n}^{q-p_{n}+2}(t)}{2 \varpi_{n}^{q-p_{n}+2}(t)}\right)} \geq 0$. Incorporate the Young's inequation, one arrives

$$
-\sum_{i=2}^{n} l_{i} \psi_{i} \operatorname{sg}_{i}\left(\zeta_{i}\right) \frac{\partial \xi_{i, c}}{\partial \hat{\Phi}} \dot{\hat{\Phi}} \leq-\kappa^{2} \gamma_{n}^{2}-\sum_{i=2}^{n} \frac{l_{i}^{2}}{4} \psi_{i}\left(\frac{\partial \xi_{i, c}}{\partial \hat{\Phi}}\right)^{2}
$$

therefore, the inequation $\Im_{n} \leq 0$ always holds. Then, we can derive that

$$
\begin{aligned}
\dot{\mathfrak{L}}_{n} \leq & -\sum_{i=1}^{n} c_{i} \tan ^{q}\left(\frac{\pi \zeta_{i}^{q-p_{i}+2}(t)}{2 \varpi_{i}^{q-p_{i}+2}(t)}\right) \psi_{i}-\sum_{i=1}^{n} a_{i} \\
& \times \tan ^{p}\left(\frac{\pi \zeta_{i}^{q-p_{i}+2}(t)}{2 \varpi_{i}^{q-p_{i}+2}(t)}\right) \psi_{i} .
\end{aligned}
$$

\section{Stability Analysis}

At this point, the main result of this paper is given in the following Theorem 1.

Theorem 1: Consider the high-order nonlinear systems (1) subject to actuator faults (2). Under Assumptions 1-2, the virtual controllers (22) and (31), the state constrained fault-tolerant controller (39), the adaption laws (40). By choosing appropriate design parameter$\mathrm{s}$, then, there exist a settling time independent of initial states such that tracking errors converge into the user-defined intervals and all signals of the closed-loop system are fixed-time stable and the time-varying state constraints will never be violated.

Proof. Consider the total high-order BLFs as

$$
\overline{\mathfrak{L}}=\sum_{i=1}^{n} \frac{2 \varpi_{i}^{q-p_{i}+2}(t)}{\pi\left(q-p_{i}+2\right)} \tan \left(\frac{\pi \zeta_{i}^{q-p_{i}+2}(t)}{2 \varpi_{i}^{q-p_{i}+2}(t)}\right) \psi_{i} .
$$

Invoking (18), (19) and (21), it yields that

$$
\begin{aligned}
\dot{\overline{\mathfrak{L}}} \leq & -\sum_{i=1}^{n} c_{i} \tan ^{q}\left(\frac{\pi \zeta_{i}^{q-p_{i}+2}(t)}{2 \varpi_{i}^{q-p_{i}+2}(t)}\right) \psi_{i}+\tilde{\Phi} \gamma_{n} \\
& -\sum_{i=1}^{n} a_{i} \tan ^{p}\left(\frac{\pi \zeta_{i}^{q-p_{i}+2}(t)}{2 \varpi_{i}^{q-p_{i}+2}(t)}\right) \psi_{i} .
\end{aligned}
$$

Substituting (45) into (58), we get

$$
\begin{aligned}
\tilde{\Phi} \gamma_{n} & =\tilde{\Phi} \sum_{i=1}^{n} \tan ^{q}\left(\frac{\pi \zeta_{i}^{q-p_{i}+2}(t)}{2 \varpi_{i}^{q-p_{i}+2}(t)}\right) \psi_{i} \sqrt{\vartheta_{i}^{T} \vartheta_{i}+\lambda_{0}} \\
& \leq \omega_{i_{m}} \sqrt{2 n\left(2+\lambda_{0}\right)} \psi_{i}^{1-q}\left(\frac{\pi\left(q-p_{i}+2\right)}{2 \varpi_{i}^{q-p_{i}+2}(t)}\right)^{q} \overline{\mathfrak{L}}^{q}
\end{aligned}
$$

where $\omega_{i_{m}}$ stands for the upper bound of estimation error $\tilde{\omega}_{i}$. Then the time derivative of $\bar{V}_{i}$ becomes

$$
\dot{\overline{\mathfrak{L}}}_{i} \leq-a_{1} \mathfrak{L}_{i}^{p}(t)-b_{1} \mathfrak{L}_{i}^{q}(t)
$$

where $a_{1}=\frac{\pi\left(q-p_{i}+2\right) a_{i}}{2 \varpi_{i}^{q-p_{i}+2}(t)}, b_{1}=\min _{i \in\{1,2, \ldots, n\}}\left\{\frac{\pi\left(q-p_{i}+2\right) c_{i}}{2 \varpi_{i}^{q-p_{i}+2}(t)}-\right.$ $\left.\omega_{i_{m}} \sqrt{2 n\left(2+\lambda_{0}\right)} \psi_{i}^{1-q}\left(\frac{\pi\left(q-p_{i}+2\right)}{2 \varpi_{i}^{q-p_{i}+2}(t)}\right)^{q}\right\}$. With the results obtained in (46), it leads to that the inequations $\mathfrak{L}_{n} \leq$ 0 hold, therefore, we can derive that $\zeta_{i}$ and $\tilde{\Phi}$ are bounded. Due to that $\zeta_{i}=\left|\xi_{i}\right|-\tau_{i}$ and the value of $\beta_{i}$ is user-defined, thus $\xi_{i}$ is bounded. Since $\hat{\Phi}=\Phi-\tilde{\Phi}$ and $\Phi$ is a constant, we have $\hat{\Phi}$ is bounded. Since $\xi_{i}$; $\mathfrak{L} \in L_{\infty}$. Therefore, all the signals of closed-loop system are bounded. In view of Lemma 2 and previous analysis, we can conclude that the tracking errors $\xi_{1}$ will asymptotically converge to $y_{r}$ within fixed-time $T=\frac{1}{a_{1}}+\frac{2}{b_{1}}$. The proof is thus completed. 

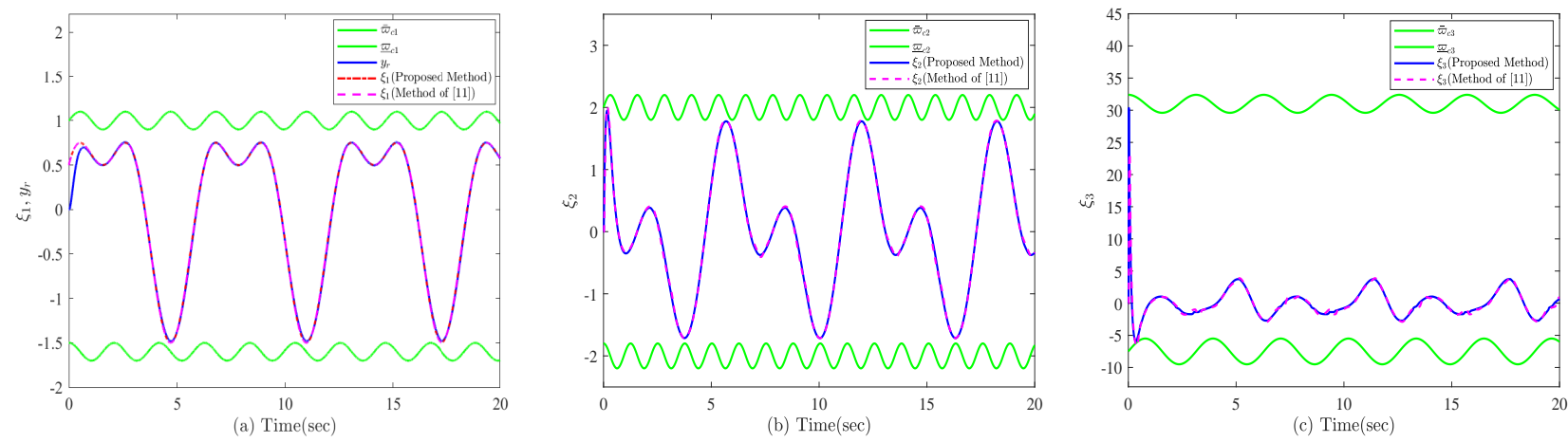

Fig. 1: (a) Evolution of $y_{1}$ and $y_{r}$, (b) Evolution of $\xi_{2}$ under two schemes, (c) Evolution of $\xi_{3}$ under two schemes.
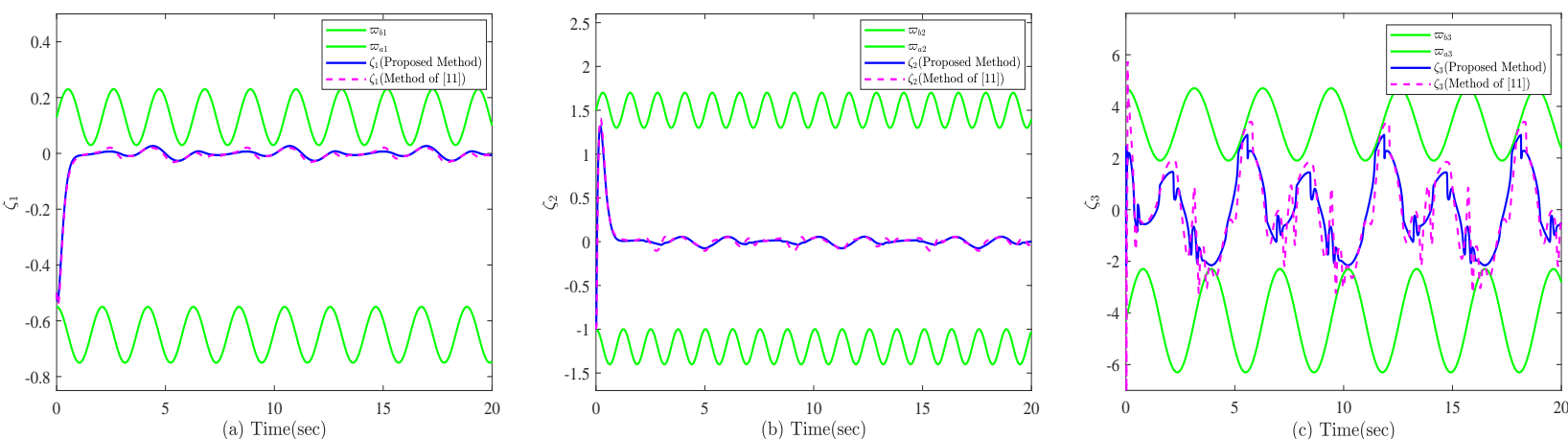

Fig. 2: (a) Profile of $\zeta_{1}$ under two schemes, (b) Profile of $\zeta_{2}$ under two schemes, (c) Profile of $\zeta_{3}$ under two schemes.

\section{Simulation Example}

To verify the effectiveness of the proposed scheme in practical application, a poppet valve system which is one of the most commonly used components in hydraulic systems is considered [1]. A poppet valve is typically utilized to control the timing and quantity of gas or vapor flow into an engine, and its behavior can be modeled by the annular leakage equation. The input force $F$ drives the poppet to move for regulating the volumetric flow rate $Q_{\text {vol }}=\lambda c^{3}$ of oil from the high-pressure to the low-pressure chamber, where $\lambda=\frac{\pi r}{6 \mu L} \Delta P$ is a lumped coefficient, $c=\alpha y$ is the effective clearance of the annular passage with $\alpha$ a constant and $y$ the displacement of poppet, and where $r, \mu$ and $L$ are constants independent of the axial motion of poppet, and $\Delta P$ is the pressure drop between two chambers. The dynamics of oil volume $V$ in upper chamber is given by

$$
\dot{V}=Q_{\mathrm{vol}}-R(t)
$$

where $R$ is the lumped reduction rate of oil attributed to consumption and other leakages. Likewise, the equation of motion of the poppet is

$$
m \ddot{y}=-k \dot{y}(t)+T(t)+F(t),
$$

where $m$ is the mass of the poppet, $k$ is the viscous friction coefficient, $T$ is the lumped elastic force, and $F$ represents the input force. At this point, let us introduce the following notation substitutions:

$$
\xi_{1}=V, \xi_{2}=y, \xi_{3}=\dot{y}, u=F,
$$

Then, the dynamic of systems (52) and (53) comes down to

$$
\dot{\xi}_{1}=\Gamma_{1} \xi_{2}^{3}+\Psi_{1}, \dot{\xi}_{2}=\xi_{3}, \dot{\xi}_{3}=\Gamma_{3} u+\Psi_{3},
$$

where $\Gamma_{1}=\lambda \alpha^{3}, \Psi_{1}=-R, \Gamma_{3}=1 / m, \Psi_{3}=\frac{1}{m}\left[T-k \xi_{3}\right]$ with $m=7.5 \mathrm{~kg}, k=2.5 \mathrm{~N} / \mathrm{m}, R=5 \mathrm{~L} / \mathrm{min}, \Delta P=$ $10 \mathrm{~N} / \mathrm{m}^{2}, T=5 \mathrm{~N}, \mu=2.5, L=5, r=1.25, \alpha=4.5$. The reference signal is $y_{r}(t)=\sin (t)+0.5 \cos (2 t)$. While conducting the simulation, the initial state values are chosen as $y_{r}(0)=0, \xi_{1}(0)=[0,0,0]^{T}$ and $\hat{\Phi}(0)=$ 0.5 , and the design parameters are chosen as $c_{1}=7.5$, $c_{2}=8.2, c_{3}=9, a_{1}=5.5, a_{2}=6, a_{3}=7, \kappa=1, o_{1}=$ $1.15, o_{2}=1.2, o_{3}=1.05, p=1.1, q=0.9, \beta_{1}=0.02$. Consider the actuator fault $u=\mathcal{H} u_{c}+\eta_{u}$ with $\mathcal{H}=$ $\left\{\begin{array}{c}1, \quad t \leq 10, \\ 0.6, t>10,\end{array}\right.$ and $\eta_{u}=\left\{\begin{array}{c}0, \quad t \leq 10, \\ 1+0.5 \sin (t), t>10,\end{array}\right.$. The system states are restrained in $\underline{\varpi}_{c i}(t)<\xi_{i}(t)<\bar{\varpi}_{c i}(t)$ with $\underline{\varpi}_{c 1}(t)=0.1 \cos (3 t)-1.6, \bar{\varpi}_{c 1}(t)=0.1 \sin (3 t)+$ $1, \underline{\varpi}_{c 2}(t)=0.2 \cos (5 t)-2, \bar{\varpi}_{c 2}(t)=0.2 \sin (5 t)+2$, $\underline{\varpi}_{c 3}(t)=2 \sin (2 t)-7.5, \bar{\varpi}_{c 3}(t)=1.4 \cos (2 t)+31$, and 


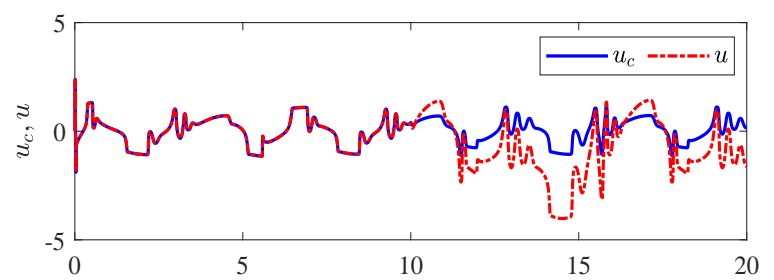

(a) Time(sec)

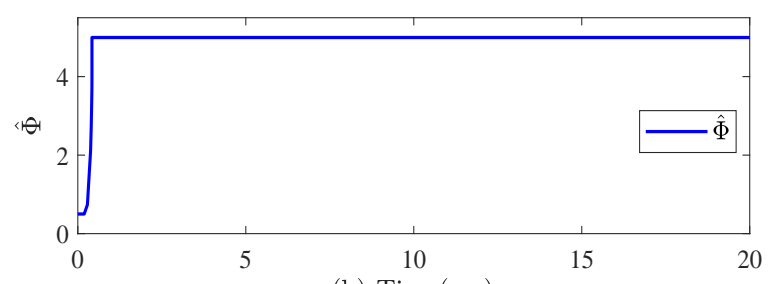

(b) Time(sec)

Fig. 3: (a) The fault tolerant controller $u_{c}$ and actual control input $u$, (b) The adaptation law $\hat{\Phi}$.

the lower and upper bound of dynamics tracking error are given as $\varpi_{b 1}(t)=0.1 \cos (3 t)-0.65, \varpi_{a 1}(t)=$ $0.1 \sin (3 t)+0.13, \varpi_{b 2}(t)=0.2 \cos (5 t)-1.2, \varpi_{a 2}(t)=$ $0.2 \sin (5 t)+1.5, \varpi_{b 3}(t)=2 \sin (2 t)-4.3, \varpi_{a 3}(t)=$ $1.4 \cos (2 t)+3.3$.

The simulation results are shown in Figs. 1-3, where the standard adaptive control scheme as in [11] is taken as a means of comparison. It can be perceived from Figs. 1-(a) and 2-(a) that the system output $y$ tracks the desired trajectory $y_{r}$ closely and the tracking error converges to a specified interval within fixed-time, and the proposed control scheme possesses a better performance than the method in [11]. Figs. 1-(b), (c) and 2 -(b), (c) show the profiles of $\xi_{2}, \xi_{3}, \zeta_{2}$ and $\zeta_{3}$. The asymmetric and time-varying constraints are guaranteed via the proposed control scheme, while the system state constraint is violated with the control method in [11]. Besides, compared with the existing results, the fluctuation of tracking error is smaller by utilizing the proposed method. The boundness of the controller and adaptive parameters is illustrated in Fig. 3. As can be seen from the above Fig. 3, the proposed controller owns good robustness to the actuator faults.

\section{Conclusions}

An asymmetric time-varying barrier Lyapunov functionsbased nonsingular fixed-time adaptive FTC method is constructed for high-order nonlinear systems in the presence of asymmetric time-varying state constraints and actuator faults. By constructing a new tuning function and a projection operator, the size of the conver- gence regions of state tracking errors in our case can be adjustable. The singularity and chatting problems are avoided by utilizing the switch function and smooth sign function.

\section{Data availability}

Data sharing is not applicable to this article as no datasets were generated or analyzed during the current study.

\section{Conflict of interest}

The authors declare that they have no conflict of interest.

\section{References}

1. Manring, N.D., and Fales, R.C.: Hydraulic Control Systems, New York, USA: John Wiley, (2019)

2. Yoo, S.J.: Low-complexity robust tracking of high-order nonlinear systems with application to underactuated mechanical dynamics. Nonlinear Dynamics. 91(3), 16271637 (2018)

3. Liu, Z., Xue, L., Sun, W., and Sun, Z.: Robust output feedback tracking control for a class of high-order timedelay nonlinear systems with input dead-zone and disturbances. Nonlinear Dynamics. 97(2), 921-935 (2019)

4. Wu, L., Park, J.H., Xie, X., Liu, Y.: Neural network adaptive tracking control of uncertain MIMO nonlinear systems with output constraints and event-triggered inputs. IEEE transactions on neural networks and learning systems. 32(2), 695-707 (2021)

5. Wu, L., Park, J.H., Xie, X., Ren, Y., Yang, Z.: Distributed adaptive neural network consensus for a class of uncertain nonaffine nonlinear multi-agent systems. Nonlinear Dynamics. 100(2), 1243-1255 (2020)

6. Qian, C., and Lin, W.: Practical output tracking of nonlinear systems with uncontrollable unstable linearization. IEEE Transactions on Automatic Control. 47(1), 21-36 (2002)

7. Lin, W., and Qian, C.: Adding one power integrator: a tool for global stabilization of high-order lower-triangular systems. Systems \& Control Letters. 39(4), 339-351 (2000)

8. Sun, Z., Li, T., and Yang, S.: A unified time varying feedback approach and its applications in adaptive stabilization of high-order uncertain nonlinear systems. Automatica. 70, 249-257 (2016)

9. Li, F., and Liu, Y.: Global practical tracking with prescribed transient performance for inherently nonlinear systems with extremely severe uncertainties. Science China Information Sciences. 62, 1-16 (2019)

10. Chen, C., and Chen, G.: A new approach to stabilization of high-order nonlinear systems with an asymmetric output constraint. International Journal of Robust and Nonlinear Control. 30, 756-775 (2020)

11. Zhao, X., Shi, P., Zheng, X., and Zhang, J.: Intelligent tracking control for a class of uncertain high-order nonlinear systems. IEEE Transactions on Neural Networks and Learning Systems. 27(9), 1976-1982 (2016) 
12. Wang, X., Li, H., Zong, G., and Zhao, X.: Adaptive fuzzy tracking control for a calss of high-order switched uncertain nonlinear systems. Journal of the Franklin Institute. 354(4), 6567-6587 (2017)

13. Shi, C., Liu, Z., Dong, X., and Chen, Y.: A novel errorcompensation control for a class of high-order nonlinear systems with input delay. IEEE Transactions on Neural Networks and Learning Systems. 29(9), 4077-4087 (2018)

14. Li, Y., Li, K., and Tong, S.: Finite-time adaptive fuzzy output feedback dynamic surface control for MIMO nonstrict feedback systems. IEEE Transactions on Fuzzy Systems. 27(1), 96-110 (2019)

15. Wang, F., Chen, B., Liu, X.P., and Lin, C.: Finite-time adaptive fuzzy tracking control design for nonlinear systems. IEEE Transactions on Fuzzy Systems. 26(3), 1207$1216(2018)$

16. Sui, S., Chen C.L.P., and Tong, S.: Fuzzy adaptive finitetime control design for nontriangular stochastic nonlinear systems. IEEE Transactions on Fuzzy Systems. 27(1), 172-184 (2019)

17. Sun, Z., Shao, Y., and Chen, C.: Fast finite-time stability and its application in adaptive control of high-order nonlinear system. Automatica. 106, 339-348 (2019)

18. Fang, L., Ma, L., Ding, S., and Park, J.H.: Finite-time stabilization of high-order stochastic nonlinear systems with asymmetric output constraints. IEEE Transactions on Systems, Man, and Cybernetics: Systems. (2020). http://doi.org/10.1109/TSMC.2020.2965589

19. Polyakov, A.: Nonlinear feedback design for fixed-time stabilization of linear control systems. IEEE Transactions on Automatic Control. 57(8), 2106-2110 (2012)

20. Cao, Y., Wen, C., Tan, S., and Song, Y.: Prespecifiable fixed-time control for a class of uncertain nonlinear systems in strict-feedback form. International Journal of Robust and Nonlinear Control. 30(3), 1203-1222 (2020)

21. Sun, Y., Wang, F., Liu, Z., Zhang, Y., and Chen, C.L.P.: Fixed-time fuzzy control for a class of nonlinear systems. IEEE Transactions on Cybernetics. (2020). http://doi.org/10.1109/TCYB.2020.3018695

22. Chen, C., and Sun, Z.: Fixed-time stabilisation for a class of high-order non-linear systems. IET Control Theory \& Applications. 18(12), 273-280 (2018)

23. Liu, Y.: Adaptive control-based barrier Lyapunov functions for a class of stochastic nonlinear systems with full state constraints. Automatica. 87, 83-93 (2018)
24. Tee, K.P.: and Ge, S.S.: Barrier lyapunov functions for the control of output-constrained nonlinear systems. Automatica. 45, 918-927 (2009)

25. Niu, B., and Zhao, J.: Barrier lyapunov functions for the output tracking control of constrained nonlinear switched systems. Systems \& Control Letters. 62(10), 963-971 (2013)

26. Liu, Y., and Tong, S.: Barrier lyapunov functions-based adaptive control for a class of nonlinear pure-feedback systems with full state constraints. Automatica. 64, 7075 (2016)

27. Wu, Y., and Xie, X.: Adaptive fuzzy control for highorder nonlinear time-delay systems with full-state constraints and input saturation. IEEE Transactions on Fuzzy Systems. 28(8), 1652-1663 (2020)

28. Xu, J.: Iterative learning control for output-constrained systems with both parametric and nonparametric uncertainties. Automatica. 49, 2508-2516 (2013)

29. Liu, L., Liu, Y., Chen, A., Tong, S., and Chen, C.L.P.: Integral barrier lyapunov function-based adaptive control for switched nonlinear systems. Science China Information Sciences. 63, 1-14 (2020)

30. Sun, Q., Fan, R., Li, Y., Huang, B., and Ma, D.: A distributed double- consensus algorithm for residential we-energy. IEEE Transactions on Industrial Informatics. 15(8), 4830-4842 (2019)

31. Sun, W., Su, S., Dong, G., and Bai, W.: Reduced adaptive fuzzy tracking control for high-order stochastic nonstric$\mathrm{t}$ feedback nonlinear system with full-state constraints. IEEE Transactions on Systems, Man, and Cybernetics: Systems. 51(3), 1496-1506 (2021)

32. Yu, Z., Liu, Z., Zhang, Y., Qu, Y., and Su, C.: Distributed finite-time fault-tolerant containment control for multiple unmanned aerial vehicles. IEEE Transactions on Neural Networks and Learning Systems. 31(6), 2077-2091 (2020)

33. Lv, M., Yu, W., Cao, J., and Baldi, S.: A separationbased methodology to consensus tracking of switched high-order nonlinear multi-agent systems. IEEE Transactions on Neural Networks and Learning Systems. (2021). http://doi.org/10.1109/TNNLS.2021.3070824

34. Lu, K., Liu, Z., Lai, G., and Chen, C.L.P.: Adaptive consensus tracking control of uncertain nonlinear multiagent systems with predefined accuracy. IEEE Transactions on Cybernetics. 51(1), 405-415 (2021) 